\title{
Desigualdades socioeconómicas en la mortalidad en Pamplona y Logroño en el periodo 1996-2007
}

\section{Socioeconomic inequalities in mortality in Pamplona and Logroño (cities in the North of Spain) in the 1996-2007 period}

\author{
N. Izco Goñi ${ }^{1}$, J. Etxeberria Andueza ${ }^{2,3}$, J. Delfrade Osinaga ${ }^{3,4}$, Y. Floristán Floristán ${ }^{3,4}$, \\ C. Moreno Iribas ${ }^{3,4}, y$ otros miembros de MEDEA*
}

\section{RESUMEN}

Fundamento. Describir las desigualdades en mortalidad en Logroño y Pamplona, ciudades del norte de España, utilizando un indicador de privación socioeconómica por secciones censales.

Métodos. Para geocodificar la residencia de los fallecidos en base a las unidades censales se utilizó la dirección postal del Boletín Estadístico de Defunción y en su defecto la del Padrón Municipal o Tarjeta Sanitaria. Las variables socioeconómicas que componen el índice de privación se obtuvieron del Censo de Población y Vivienda de 2001. Se agruparon las secciones censales por quintiles de menor a mayor privación socioeconómica y se calcularon tasas ajustadas para la mortalidad total y para una lista de causas por sexo, grupo de edad (0-64, $\geq 65$ ) y quintil del índice de privación socioeconómico.

Resultados. La comparación de la mortalidad de las zonas con menor privación socioeconómica respecto a zonas geográficas con mayor privación mostró en ambas ciudades una mayor mortalidad principalmente entre los hombres menores de 65 años. En este subgrupo se observó un gradiente positivo entre la mortalidad y el índice de privación. Las zonas situadas en el quintil de mayor privación alcanzaron un riesgo relativo (RR) de 1,61 (IC 95\%, 1,33-1,92) en Pamplona y de 1,77 (IC 95\%, 1,55-2,01) en Logroño. Las mujeres menores de 65 años mostraron un RR de 1,44 (IC $95 \%, 1,18-1,74$ ) y de $1,48$ (IC $95 \%, 1,10-1,95)$ en las zonas situadas en el quintil de mayor privación de Pamplona y Logroño, respectivamente.

Conclusiones. El estudio evidenció desigualdades en mortalidad asociadas a la privación socioeconómica en las ciudades de Logroño y Pamplona. También mostró la utilidad de los indicadores socioeconómicos y de mortalidad a nivel de área pequeña para identificar desigualdades en salud.

Palabras clave. Mortalidad. Factores socioeconómicos. Análisis de áreas pequeñas. MEDEA.

An. Sist. Sanit. Navar. 2013; 36 (2): 229-240

\begin{abstract}
Background. The objective of this study was to describe inequalities in mortality in the cities of Logroño and Pamplona, using a socioeconomic privation index by census tract.

Methods. Deaths were geocoded using the mailing address of the Death Statistics Bulletin and, in its absence, that of the Spanish Municipal Register or the Spanish Health Card. The socioeconomic variables used to construct the deprivation index were obtained from the Spanish Population and Housing Census of 2001. Census tracts were grouped by socioeconomic deprivation quintiles. In each quintile, age-adjusted mortality rates were computed by sex and age group $(0-64, \geq 65)$.
\end{abstract}

Results. In both cities, higher mortality rates were observed in the geographical areas with higher socio-economic deprivation. This was particularly evident among men under 65 for whom a positive gradient between mortality and the deprivation index was observed. The areas with higher deprivation reached a relative risk (RR) of 1.61 (CI 95\%, 1 , 33-1, 92) and 1.77 (CI 95\%, 1, 55-2 01) in Pamplona and Logroño respectively. Among women under 65, a RR of 1.44 (Cl $95 \%, 1,18-1,74)$ and 1.48 (CI 95\%, 1, 10-1, 95) were shown for the most depressed areas of Pamplona and Logroño respectively.

Conclusions. The study highlighted inequalities in mortality associated with socioeconomic deprivation in the cities of Logroño and Pamplona. Also showed was the utility of analysing socio-economic indicators and mortality by small areas in order to identify inequality in health.

Key words. Mortality. Socioeconomic factors. Small area analysis. MEDEA

\section{Correspondencia:}

Conchi Moreno Iribas

Instituto de Salud Pública y Laboral de Navarra Leyre 15

31003 Pamplona (Navarra)

E-mail: mmorenoi@cfnavarra.es

Financiación: ISCIII (PI08-1058) e ISCIII (PI08-1785)

Recepción: 16 de enero de 2013

Aceptación provisional: 15 de febrero de 2013

Aceptación definitiva: 8 de mayo de 2013 


\section{INTRODUCCIÓN}

Actualmente las desigualdades en salud suponen un importante problema para los países desarrollados y se relacionan directamente con las desigualdades sociales. En los estudios de desigualdades en salud, los indicadores de mortalidad han sido muy utilizados por ser una de las fuentes de información más accesibles y comparables a nivel local o global. Existen muchos estudios destinados a evaluar desigualdades en mortalidad en asociación con indicadores sociales y económicos ${ }^{1,2}$. Las poblaciones con niveles socioeconómicos menos favorecidos presentan peores indicadores de salud $^{3}$ y mayores tasas de mortalidad ${ }^{4}$ para la mayoría de las causas de muerte, en particular para las enfermedades cardiovasculares y algunos tipos de cáncer ${ }^{5}$.

La evaluación de la salud y los indicadores socioeconómicos de privación a nivel de área pequeña permiten identificar gradientes socioeconómicos en salud. Los resultados obtenidos en este contexto son comparables o incluso mejores que los obtenidos en aquellos estudios en los que se evalúan indicadores individuales ${ }^{6-9}$. Los estudios por áreas geográficas son importantes por varias razones. En primer lugar, existen factores contextuales a nivel de zona que explican diferencias en salud: el entorno físico, el crecimiento urbano, el mercado de trabajo, las instalaciones de ocio, los centros educativos, centros sanitarios o sociales, entre $\operatorname{otros}^{10,11}$. En segundo lugar, la identificación de áreas geográficas con peor salud y peores condiciones socioeconómicas facilita la implementación de las intervenciones y las políticas para combatir las desigualdades ${ }^{12}$. Finalmente, monitorizar las desigualdades en salud utilizando datos ecológicos puede ser más factible que emplear datos a nivel individual.

El proyecto "Mortalidad en áreas pequeñas Españolas y Desigualdades Socioeconómicas y Ambientales (MEDEA)" es un proyecto coordinado entre 14 grupos de investigación, que tiene como propósito estudiar las desigualdades socioeconómicas y medioambientales en áreas pequeñas de ciudades de España. La primera fase del estudio se desarrolló empleando datos de mortalidad entre 1996-2003, y se evidenció que en la mayoría de las 11 ciudades participantes y para ambos sexos, existían similitudes entre el patrón geográfico de mortalidad y el patrón geográfico de privación socioeconómica ${ }^{13}$, es decir, a mayor privación mayor mortalidad. Logroño y Pamplona no participaron en la primera edición del estudio, se incorporaron en la $2^{\circ}$ fase en la cual hubo 34 ciudades participantes y se amplió el periodo de estudio hasta 2007.

Se planteó como objetivo de este trabajo describir las desigualdades socioeconómicas en la mortalidad total y por causas específicas en áreas pequeñas de las ciudades de Pamplona y Logroño, dos ciudades del norte de España, en el periodo 1996-2007.

\section{MÉTODOS}

El presente estudio de diseño ecológico analizó las desigualdades en la mortalidad total y por causas específicas en las ciudades de Pamplona y Logroño en el período de 1996-2007 tomando como unidad de análisis la sección censal. Cada unidad censal se compone de 1.000 habitantes aproximadamente y el número de secciones censales consideradas fueron 122 en Pamplona y 91 en Logroño. Para el análisis de los datos de Logroño no se incluyó la sección 20 del distrito 2, por la alta concentración de residencias de ancianos en esa sección.

Para la evaluación de la desigualdad entre las distintas unidades de análisis se consideró un índice de privación socioeconómica asociado a cada sección censal de residencia. El índice utilizado en este estudio se obtuvo a partir de indicadores socioeconómicos disponibles en el Censo de Población y Vivienda de 2001 utilizando la metodología desarrollada por Domínguez Berjón y col ${ }^{14}$. Brevemente, mediante el análisis de componentes principales se calculó un índice de privación que combinaba información de cinco indicadores socioeconómicos: (a) Desempleo: porcentaje de personas de 16 o más años buscando empleo en relación a la población total económicamente activa; (b) Bajo nivel educacional: porcentaje de población 
de 16 años o más con menos de 5 años de escolarización o con 5 o más años de escolarización que no completaron los estudios básicos en relación a la población total de 16 o más años; (c) Bajo nivel de educación en jóvenes (16-29 años); (d) Trabajadores manuales: porcentaje de personas de 16 o más años, ocupadas, que son trabajadores manuales en relación a la población total empleada de 16 o más años y (e) Trabajadores eventuales: porcentaje de población de 16 o más años, empleados en trabajos temporales, en relación a la población total empleada de 16 ó más años.

Este índice recogía, según las ciudades, el $75,9-85,6 \%$ de la variabilidad de los indicadores que lo componían. El índice se normalizó con media 0 y desviación típica 1. Refleja la privación material de cada sección censal: cuanto mayor es el índice, mayor es su privación. Los niveles de privación no son directamente comparables entre ciudades. Destacar que, el índice propuesto por Dominguez-Berjón y $\mathrm{col}^{14}$, añade algún indicador nuevo respecto a los utilizados en otros estudios realizados en España, en concreto el referido a los asalariados eventuales y el de instrucción insuficiente en jóvenes. Los autores consideraron importante incorporar estas variables para caracterizar mejor la privación socioeconómica en la sociedad actual.

\section{Fuente de datos}

Los datos sobre las defunciones de residentes de Pamplona y Logroño ocurridas en España en el periodo 1996-2007 provinieron de los registros de Mortalidad de Navarra y de La Rioja, los datos de poblaciones del Instituto Nacional de Estadística (INE) y las variables con las que se elaboró el índice de privación socioeconómico del Censo de Población y Vivienda de 2001. Los proyectos fueron aprobados por los Comités Éticos de Investigación Clínica de Navarra y La Rioja.

\section{Geocodificación de las defunciones}

La asignación del distrito y la sección censal se realizó de forma manual a partir de la dirección postal del Boletín Estadísti- co de Defunción (BED). El callejero utilizado fue el del censo electoral del año 2001 facilitado por el Instituto Nacional de Estadística. Se tuvieron en cuenta los cambios producidos en los códigos de sección de los callejeros y se reasignaron las defunciones afectadas.

En Logroño, en una primera fase se codificaron aquellos registros que proporcionaban una dirección de residencia completa, el $93 \%$ de un total de 13.375 casos. La información del $7 \%$ restante se completó con la proveniente de fuentes alternativas como la base de datos de la Tarjeta Sanitaria, SELENE (base de datos del Hospital San Pedro de Logroño) y Padrón Continuo de Población. También se dispuso de la ayuda del Área de Estadística del Ayuntamiento de Logroño. Finalmente se alcanzó la geocodificación en el 99,9\% de los casos. Se encontraron 123 BEDs (0,91\%) que correspondían a direcciones de otros municipios.

En Pamplona, se encontró una coincidencia total entre la dirección del BED y el callejero del censo electoral del año 2001 para 16.744 casos sobre un total de 19.274. Los BEDs restantes, 2.530, presentaban una casuística diferente: direcciones incompletas o con errores en los datos de residencia: calles que no correspondían a Pamplona sino a otros municipios de Navarra e incluso otras ciudades. En estos casos se buscó información adicional en la base de datos de Tarjeta Sanitaria y el Censo de 1986. Finalmente se alcanzó la geocodificación en el $98,9 \%$ de los casos. Señalar que se encontraron 247 BEDs que correspondían a otros municipios de Navarra y 14 a otras ciudades de fuera de Navarra.

\section{Análisis estadístico}

Se realizó una agrupación de las secciones censales por quintiles de privación. En el primer quintil se agruparon las secciones censales con menor privación socioeconómica y en el último quintil aquellas secciones con mayor privación socioeconómica. En cada quintil se computaron el número total de defunciones observadas, las tasas de mortalidad estandarizadas por edad según el método directo (población 
europea) y los años de esperanza de vida al nacimiento y con 1, 15, 45 y 65 años de edad, utilizando la tabla de vida abreviada según el método de Chiang ${ }^{15}$. La esperanza de vida a la edad $\mathrm{x}\left(\mathrm{e}_{\mathrm{x}}\right)$ representa el número medio de años que a un individuo de edad $\mathrm{x}$ perteneciente a la cohorte ficticia inicial le restaría por vivir. Su valor resulta del cociente entre el tiempo total (medido en años) que le resta por vivir a partir de cumplir $\mathrm{x}$ años de edad a los individuos de la generación ficticia hasta su completa extinción y el número de supervivientes de la misma a la edad $\mathrm{x}$ :

$$
\begin{gathered}
\mathrm{e}_{\mathrm{x}}=\sum_{\mathrm{y} \geq \mathrm{x}} \mathrm{L}_{\mathrm{y}} / \mathrm{l}_{\mathrm{x}} \\
\mathrm{l}_{\mathrm{x}} \text { son los supervivientes a la edad } \mathrm{x} \\
\mathrm{L}_{\mathrm{y}} \text { es la población estacionaria a la edad y }
\end{gathered}
$$

Para ilustrar el impacto del índice de privación en la mortalidad total y por causas específicas se empleó un modelo jerárquico bayesiano propuesto por Besag y col $^{16}$ (modelo BYM). Este tipo de modelos tiene en cuenta dos tipos de variabilidad, la estructurada y la no estructurada, además de incluir la variable índice de privación categorizada en quintiles. El modelo puede expresarse de la siguiente forma:

$$
\begin{aligned}
& \mathrm{O}_{i} \sim \operatorname{Poisson}\left(E_{i} r_{i}\right) \\
& \log \left(r_{i}\right)=\alpha+S_{i}+H_{i}+\sum_{j=2}^{5} \beta_{i} X_{j i}
\end{aligned}
$$

donde $O_{i}$ is el número de casos observados, $E_{i}$ el número de casos esperados y $r_{i}$ es el riesgo relativo del área $i$ respecto al global de la ciudad, $S_{i}$ es el efecto espacial y $H_{i}$ es el efecto heterogéneo. El efecto aleatorio espacial permite explicar la dependencia espacial entre las áreas o dicho de otro modo, permite que los riesgos relativos de las áreas vecinas sean similares. El efecto aleatorio heterogéneo permite explicar la variabilidad de los datos debida a variables no relacionadas con el área. En la expresión anterior, $X_{j i}$ toma el valor $1 \mathrm{si}$ la sección censal i-ésima que pertenece al quintil j del índice de privación, y 0 en caso contrario. $\operatorname{Exp}\left(\beta_{j}\right)$ es el riesgo relativo (RR) de mortalidad de las secciones en el quintil j del índice de privación con respecto al primer quintil, el que presenta la mas baja privación socioeconómica. $\operatorname{Exp}\left(\beta_{5}\right)$ permi- te calcular el riesgo relativo de las secciones censales situadas en el último quintil en relación a las situadas en el primer quintil. Las estimaciones de RR fueron calculados a través de la media de la distribución a posteriori y su intervalo de credibilidad al 95\% (IC 95\%) obtenidos a partir del modelo espacial anterior. El intervalo de credibilidad al 95\% del riesgo relativo nos indica que hay una probabilidad del $95 \%$ de que el verdadero valor del riesgo se encuentre en dicho intervalo.

La causa básica de defunción fue codificada mediante la novena revisión de la Clasificación Internacional de Enfermedades (CIE-9) en el periodo 1996-1998 y con la décima revisión (CIE-10) entre 1999-2007. Se estudió la mortalidad por todas las causas y por las principales causas de muerte: cáncer de pulmón (CIE-9 162, CIE-10 C33C34), cáncer de mama en mujeres (CIE-9 174-175, CIE-10 C50), cáncer de estómago (CIE-9 151, CIE-10 C16), cáncer de colon y recto (CIE-9 153-154, CIE-10 C18-C21), cáncer de próstata (CIE-9 185, CIE-10 C61), sida (CIE-9 279.5-279.6, 795.8, CIE-10 B20-B24, R75), diabetes (CIE-9 250, CIE-10 E10-E14), cardiopatía isquémica (CIE-9 410-414, CIE10 I20-I25), enfermedad cerebrovascular (CIE-9 430-438, CIE-10 I60-I69), enfermedad pulmonar obstructiva crónica (EPOC, CIE9 490-496, CIE-10 J40-J47), cirrosis hepática (CIE-9 571, CIE-10 K70, K72.1, K73-K74, K76.1, K76.9), accidentes de tráfico (CIE-9 810-819, CIE-10 V01-V99), sobredosis (CIE-9 850-869, CIE-10 X40-X49), caídas accidentales (CIE9 880-886, 888, CIE-10 W00-W19) y suicidio (CIE-9 950-959, CIE-10 X60-X84). Las causas con menos de 2 casos en el primer o quinto quintil fueron excluidos para el análisis de los riesgos relativos. Todo el análisis se realizó por separado para hombres y mujeres en dos grupos de edad ( $0-64 \mathrm{y} \geq 65$ años).

\section{RESULTADOS}

La figura 1 muestra de color gris y rojo las unidades censales de menor y mayor privación socioeconómica de las ciudades de Pamplona y Logroño. En Pamplona las secciones censales con mayor privación se sitúan principalmente al norte de la ciu- 
dad, mientras que las más favorecidas se encuentran en la parte sur-oeste. En el caso de Logroño se observa un patrón norte-sur de manera que la mayoría de las unidades censales con situación socioeconómica desfavorable se encuentran en el norte de la ciudad aunque también se encuentran un par de ellas al este de la misma.
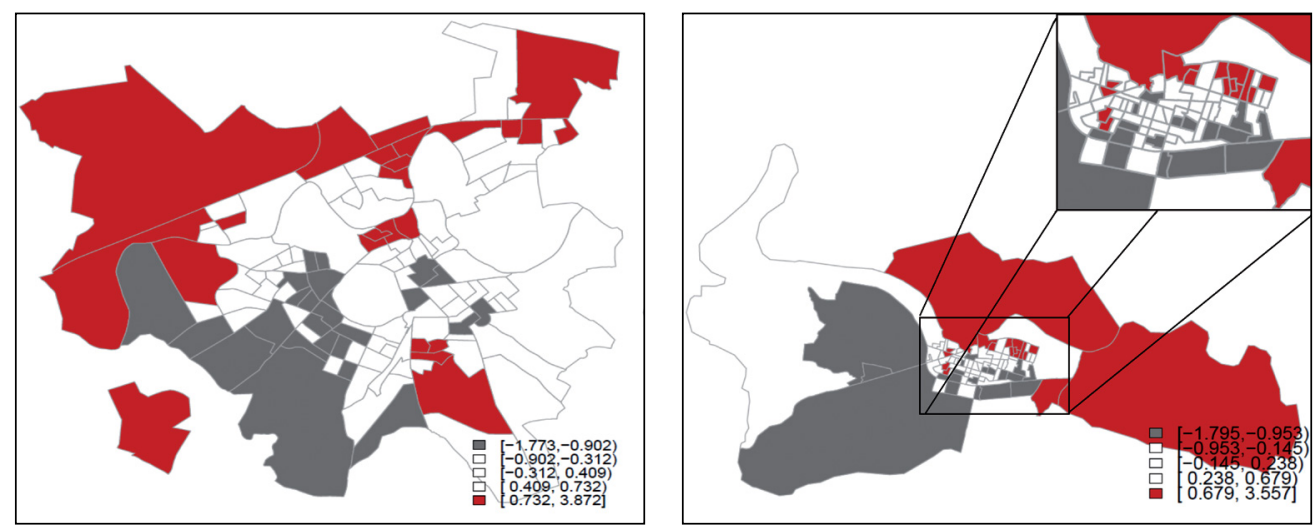

Figura 1. Distribución de las unidades censales según privación socioeconómica de Pamplona (figura izq.) y Logroño (figura dcha.) en 2001. De color gris se indican las secciones agrupadas en el quintil con menor privación y en rojo las de mayor privación.

Tabla 1. Número de defunciones, tasas estandarizadas por edad y razón de tasas de mortalidad según el índice de privación socioeconómico del lugar de residencia de Pamplona y Logroño (1996-2007).

\begin{tabular}{|c|c|c|c|c|c|c|c|c|c|}
\hline \multirow{3}{*}{$\begin{array}{c}\text { Edad } \\
\text { (años) }\end{array}$} & \multirow{3}{*}{$\begin{array}{c}\text { Quintiles } \\
\text { del índice } \\
\text { socioeconómico }\end{array}$} & \multicolumn{8}{|c|}{ Pamplona } \\
\hline & & \multicolumn{4}{|c|}{ Hombres } & \multicolumn{4}{|c|}{ Mujeres } \\
\hline & & $\begin{array}{l}\text { Número de } \\
\text { defunciones }\end{array}$ & $\begin{array}{c}\text { Tasa } \\
\text { estandar* }\end{array}$ & $\mathbf{R R}$ & (IC 95\%) & $\begin{array}{l}\text { Número de } \\
\text { defunciones }\end{array}$ & $\begin{array}{c}\text { Tasa } \\
\text { estandar* }\end{array}$ & $\mathbf{R R}$ & (IC 95\%) \\
\hline \multicolumn{10}{|c|}{ 0-64 años } \\
\hline & 1 (más rico) & 371 & 175,3 & 1,00 & - & 210 & 88,9 & 1,00 & - \\
\hline & 2 & 378 & 223,4 & 1,23 & $(1,05-1,43)$ & 168 & 92,4 & 0,99 & $(0,80-1,19)$ \\
\hline & 3 & 430 & 253,8 & 1,36 & $(1,14-1,59)$ & 194 & 103,7 & 1,21 & $(0,99-1,45)$ \\
\hline & 4 & 404 & 252,6 & 1,35 & $(1,12-1,60)$ & 183 & 111,0 & 1,18 & $(0,96-1,42)$ \\
\hline & 5 (más pobre) & 525 & 286,4 & 1,61 & $(1,33-1,92)$ & 231 & 132,5 & 1,44 & $(1,18-1,74)$ \\
\hline \multicolumn{10}{|c|}{$\geq 65$ años } \\
\hline & 1 (más rico) & 1204 & 3887,4 & 1,00 & - & 1355 & 2273,0 & 1,00 & - \\
\hline & 2 & 1756 & 4855,3 & 1,18 & $(1,06-1,31)$ & 1998 & 2370,6 & 0,95 & $(0,85-1,05)$ \\
\hline & 3 & 1604 & 4561,9 & 1,17 & $(1,05-1,30)$ & 1718 & 2337,8 & 0,99 & $(0,89-1,10)$ \\
\hline & 4 & 1363 & 4838,7 & 1,23 & $(1,10-1,37)$ & 1420 & 2508,6 & 1,07 & $(0,95-1,20)$ \\
\hline & 5 (más pobre) & 1447 & 4776,2 & 1,23 & $(1,10-1,37)$ & 1440 & 2729,3 & 1,11 & $(0,97-1,25)$ \\
\hline \multicolumn{10}{|c|}{ Todas las edades } \\
\hline & 1 (más rico) & 1575 & 583,7 & 1,00 & - & 1565 & 329,1 & 1,00 & - \\
\hline & 2 & 2134 & 732,9 & 1,21 & $(1,10-1,33)$ & 2166 & 343,0 & 0,96 & $(0,87-1,06)$ \\
\hline & 3 & 2034 & 727,7 & 1,22 & $(1,12-1,35)$ & 1912 & 349,5 & 1,02 & $(0,92-1,12)$ \\
\hline & 4 & 1767 & 757,1 & 1,28 & $(1,16-1,42)$ & 1603 & 374,7 & 1,09 & $(0,98-1,20)$ \\
\hline & 5 (más pobre) & 1972 & 780,3 & 1,34 & $(1,21-1,48)$ & 1671 & 418,2 & 1,16 & $(1,04-1,29)$ \\
\hline
\end{tabular}




\begin{tabular}{|c|c|c|c|c|c|c|c|c|c|}
\hline \multirow{3}{*}{$\begin{array}{l}\text { Edad } \\
\text { (años) }\end{array}$} & \multirow{3}{*}{$\begin{array}{c}\text { Quintiles } \\
\text { del índice } \\
\text { socioeconómico }\end{array}$} & \multicolumn{8}{|c|}{ Logroño } \\
\hline & & \multicolumn{4}{|c|}{ Hombres } & \multicolumn{4}{|c|}{ Mujeres } \\
\hline & & $\begin{array}{l}\text { Número de } \\
\text { defunciones }\end{array}$ & $\begin{array}{c}\text { Tasa } \\
\text { estandar* }\end{array}$ & $\mathbf{R R}$ & (IC 95\%) & $\begin{array}{l}\text { Número de } \\
\text { defunciones }\end{array}$ & $\begin{array}{c}\text { Tasa } \\
\text { estandar* }\end{array}$ & $\mathbf{R R}$ & (IC 95\%) \\
\hline \multicolumn{10}{|c|}{ 0-64 años } \\
\hline & 1 (más rico) & 236 & 175,3 & 1,00 & - & 120 & 78,7 & 1,00 & - \\
\hline & 2 & 306 & 228,1 & 1,33 & $(1,06-1,66)$ & 114 & 77,5 & 0,92 & $(0,68-1,22)$ \\
\hline & 3 & 284 & 228,1 & 1,33 & $(1,05-1,65)$ & 105 & 78,7 & 0,92 & $(0,68-1,22)$ \\
\hline & 4 & 300 & 240,4 & 1,40 & $(1,11-1,74)$ & 127 & 97,8 & 1,21 & $(0,90-1,60)$ \\
\hline & 5 (más pobre) & 416 & 313,5 & 1,77 & $(1,55-2,01)$ & 156 & 122,5 & 1,48 & $(1,23-1,76)$ \\
\hline \multicolumn{10}{|c|}{$\geq 65$ años } \\
\hline & 1 (más rico) & 758 & 4436,4 & 1,00 & - & 750 & 2272,7 & 1,00 & - \\
\hline & 2 & 1132 & 4645,5 & 1,04 & $(0,92-1,16)$ & 1212 & 2600,0 & 1,12 & $(0,97-1,29)$ \\
\hline & 3 & 1140 & 4109,1 & 0,92 & $(0,82-1,03)$ & 1237 & 2500,0 & 1,06 & $(0,91-1,22)$ \\
\hline & 4 & 1000 & 4472,7 & 1,03 & $(0,91-1,15)$ & 897 & 2372,7 & 1,07 & $(0,92-1,23)$ \\
\hline & 5 (más pobre) & 1065 & 4609,1 & 1,04 & $(0,97-1,12)$ & 1071 & 2600,0 & 1,14 & $(1,05-1,23)$ \\
\hline \multicolumn{10}{|c|}{ Todas las edades } \\
\hline & 1 (más rico) & 994 & 644,0 & 1,00 & - & 870 & 320,0 & 1,00 & - \\
\hline & 2 & 1438 & 714,0 & 1,13 & $(1,01-1,26)$ & 1326 & 355,0 & 1,07 & $(0,94-1,22)$ \\
\hline & 3 & 1424 & 655,0 & 1,02 & $(0,91-1,14)$ & 1342 & 345,0 & 1,04 & $(0,94-1,18)$ \\
\hline & 4 & 1300 & 706,0 & 1,12 & $(0,99-1,26)$ & 1024 & 348,0 & 1,08 & $(0,94-1,23)$ \\
\hline & 5 (más pobre) & 1481 & 786,0 & 1,21 & $(1,07-1,36)$ & 1227 & 395,0 & 1,14 & $(0,99-1,30)$ \\
\hline
\end{tabular}

RR: riesgo relativo o razón de tasas de mortalidad ajustada por edad; IC: intervalo de credibilidad

* Por 100.000 habitantes, ajustada por edad según la población europea estándar

La tabla 1 muestra, para ambas ciudades, el número de defunciones y las tasas de mortalidad estandarizadas por quintil de privación socioeconómica por grupos de edad y sexo. Se proporcionan también los riesgos relativos de mortalidad para cada quintil del índice de privación tomando como referencia el de menor privación socioeconómica. En ambas ciudades, las tasas, así como los RR, reflejan un aumento de la mortalidad en relación al aumento de la privación sobre todo entre los hombres menores de 65 años. En este grupo, el RR del quintil más desfavorecido fue de 1,61 (IC 95\%, 1,33-1,92) en Pamplona y de 1,77 (IC 95\%, 1,55-2,01) en Logroño. Entre las mujeres de 0-64 años no se observaron diferencias estadísticamente significativas en los cuatro primeros quintiles pero sí en el último quintil donde se observó un RR de 1,44 en Pamplona (IC 95\%, 1,18-1,74) y de 1,48 en Logroño (IC 95\%, 1,23-1,76).

En hombres mayores de 65 años de Pamplona se observó un gradiente posi- tivo entre la mortalidad total y el índice socioeconómico. En las mujeres de Logroño, la mortalidad presentó una asociación positiva en el quinto quintil, con un RR de 1,14 (IC 95\%, 1,05-1,23). Dado que la geocodificación se aproxima al $100 \%$ en ambas ciudades se pueden comparar las tasas de mortalidad de las zonas con mayor y menor privación socioeconómica de ambas ciudades encontrándose, como se observa en la tabla 1, una gran similitud. Entre los hombres menores de 65 años residentes en las zonas con menor privación las tasas son similares en ambas ciudades (175 por 100.000) e igualmente muy similares en las de mayor privación (313 por 100.000 en Logroño y 286 por 100.000 en Pamplona). Entre las mujeres, la mortalidad se sitúa en 88,9 y 78,7 por 100.000 en el quintil más favorecido y en 132,5 y 122,5 en las zonas más desfavorecidas de Pamplona y Logroño.

La esperanza de vida al nacimiento mostró un claro gradiente socioeconómico 
en ambos sexos. Los hombres residentes en las secciones más favorecidas de Pamplona y Logroño tuvieron una esperanza de vida al nacimiento 3,8 y 3,3 años mayor que los residentes en las zonas de mayor privación (Tabla 2). Esa diferencia dismi- nuía al aumentar la edad, hasta llegar a 1,8 y 0,4 años a los 65 años. En las mujeres, la diferencia en la esperanza de vida al nacimiento era de 2,7 y 2,2 años, que se reducía hasta 1,5 y 1,05 a los 65 años de edad.

Tabla 2. Esperanza de vida según el quintil del índice de privación socioeconómico de las secciones censales de Pamplona y Logroño (1996-2007)

\begin{tabular}{|c|c|c|c|c|c|c|c|c|c|c|}
\hline \multicolumn{11}{|c|}{ Pamplona } \\
\hline \multirow{2}{*}{$\begin{array}{l}\text { Quintiles del índice } \\
\text { socioeconómico }\end{array}$} & \multicolumn{5}{|c|}{ Hombres } & \multicolumn{5}{|c|}{ Mujeres } \\
\hline & $\begin{array}{c}\mathrm{Al} \\
\text { nacimiento }\end{array}$ & $\begin{array}{c}1 \\
\text { año }\end{array}$ & $\begin{array}{c}15 \\
\text { años }\end{array}$ & $\begin{array}{c}45 \\
\text { años }\end{array}$ & $\begin{array}{c}65 \\
\text { años }\end{array}$ & $\begin{array}{c}\mathrm{Al} \\
\text { nacimiento }\end{array}$ & $\begin{array}{c}1 \\
\text { año }\end{array}$ & $\begin{array}{c}15 \\
\text { años }\end{array}$ & $\begin{array}{c}45 \\
\text { años }\end{array}$ & $\begin{array}{c}65 \\
\text { años } \\
\end{array}$ \\
\hline 1 (más rico) & 80,0 & 79,4 & 65,5 & 36,5 & 19,0 & 86,2 & 85,3 & 71,4 & 42,1 & 23,6 \\
\hline 2 & 77,4 & 76,7 & 63,0 & 34,2 & 17,2 & 85,5 & 84,9 & 71,2 & 41,7 & 23,2 \\
\hline 3 & 77,3 & 76,5 & 62,6 & 34,4 & 17,7 & 85,7 & 84,9 & 71,0 & 41,7 & 23,4 \\
\hline 4 & 77,0 & 76,5 & 62,6 & 33,9 & 17,3 & 84,8 & 84,0 & 70,3 & 41,1 & 22,8 \\
\hline 5 (más pobre) & 76,2 & 75,5 & 61,7 & 33,7 & 17,2 & 83,5 & 83,1 & 69,3 & 40,2 & 22,1 \\
\hline Total & 77,5 & 76,9 & 63,1 & 34,5 & 17,6 & 85,2 & 84,4 & 70,7 & 41,4 & 23,0 \\
\hline $\begin{array}{l}\text { Diferencia entre } \\
\text { quintiles 1-5 }\end{array}$ & 3,8 & 3,8 & 3,7 & 2,8 & 1,8 & 2,6 & 2,2 & 2,1 & 1,9 & 1,5 \\
\hline \multicolumn{11}{|c|}{ Logroño } \\
\hline \multirow{2}{*}{$\begin{array}{l}\text { Quintiles del índice } \\
\text { socioeconómico }\end{array}$} & \multicolumn{5}{|c|}{ Hombres } & \multicolumn{5}{|c|}{ Mujeres } \\
\hline & $\begin{array}{c}\mathrm{Al} \\
\text { nacimiento }\end{array}$ & $\begin{array}{c}1 \\
\text { año }\end{array}$ & $\begin{array}{c}15 \\
\text { años }\end{array}$ & $\begin{array}{c}5 \\
\text { años }\end{array}$ & $\begin{array}{c}65 \\
\text { años }\end{array}$ & Al nacimiento & $\begin{array}{c}1 \\
\text { año }\end{array}$ & $\begin{array}{c}15 \\
\text { años }\end{array}$ & $\begin{array}{c}45 \\
\text { años }\end{array}$ & $\begin{array}{c}65 \\
\text { años }\end{array}$ \\
\hline 1 (más rico) & 79,1 & 78,3 & 64,5 & 35,6 & 17,9 & 86,3 & 85,5 & 71,5 & 42,2 & 23,5 \\
\hline 2 & 77,7 & 77,1 & 63,3 & 34,7 & 17,6 & 85,4 & 84,5 & 70,5 & 40,9 & 22,3 \\
\hline 3 & 78,5 & 77,9 & 64,0 & 35,4 & 18,5 & 85,6 & 84,8 & 71,0 & 41,5 & 22,7 \\
\hline 4 & 77,7 & 76,8 & 63,1 & 34,6 & 17,8 & 85,7 & 84,8 & 70,9 & 41,5 & 23,2 \\
\hline 5 (más pobre) & 75,8 & 75,2 & 61,6 & 33,4 & 17,5 & 84,1 & 83,6 & 69,8 & 40,6 & 22,5 \\
\hline Total & 77,7 & 77,1 & 63,3 & 34,7 & 17,9 & 85,3 & 84,6 & 70,7 & 41,3 & 22,8 \\
\hline $\begin{array}{l}\text { Diferencia entre } \\
\text { quintiles 1-5 } \\
\end{array}$ & 3,3 & 3,1 & 2,9 & 2,2 & 0,4 & 2,1 & 1,8 & 1,7 & 1,5 & 1,0 \\
\hline
\end{tabular}

En la tabla 3 se muestra el número de defunciones de cada causa específica según quintil de privación, sexo y ciudad. La tabla 4 muestra el RR de mortalidad del $5^{\circ}$ quintil respecto al primero tras excluir del análisis las causas con menos de 2 fallecidos en alguno de los quintiles de comparación (en gris, en la tabla 3). 
Tabla 3. Número de defunciones por causa y quintil del índice de privación socioeconómico de Pamplona y Logroño (1996-2007)

Pamplona

\begin{tabular}{|c|c|c|c|c|c|c|c|c|c|c|c|c|c|c|c|c|c|c|c|c|}
\hline \multirow{3}{*}{$\begin{array}{l}\text { Quintiles del índice } \\
\text { socioeconómico }\end{array}$} & \multicolumn{10}{|c|}{ Hombres } & \multicolumn{10}{|c|}{ Mujeres } \\
\hline & \multicolumn{5}{|c|}{$\leq 65$ años } & \multicolumn{5}{|c|}{ > 65 años } & \multicolumn{5}{|c|}{$\leq 65$ años } & \multicolumn{5}{|c|}{$>65$ años } \\
\hline & Q1 & Q2 & Q3 & Q4 & Q5 & Q1 & Q2 & Q3 & Q4 & Q5 & Q1 & Q2 & Q3 & Q4 & Q5 & Q1 & Q2 & Q3 & Q4 & Q5 \\
\hline Total & 371 & 378 & 430 & 404 & 525 & 1204 & 1756 & 1604 & 1363 & 1447 & 210 & 168 & 194 & 183 & 231 & 1355 & 1998 & 1718 & 1420 & 1440 \\
\hline Cáncer pulmón & 70 & 43 & 51 & 44 & 84 & 88 & 108 & 137 & 97 & 119 & 8 & 15 & 17 & 13 & 21 & 19 & 16 & 13 & 20 & 11 \\
\hline Cáncer mama & 0 & 0 & 0 & 0 & 0 & 1 & 1 & 0 & 0 & 0 & 29 & 25 & 33 & 24 & 21 & 43 & 41 & 34 & 40 & 34 \\
\hline Cáncer estómago & 9 & 16 & 11 & 12 & 17 & 26 & 38 & 42 & 38 & 31 & 5 & 4 & 10 & 4 & 4 & 19 & 24 & 34 & 28 & 18 \\
\hline Cáncer colorrectal & 17 & 19 & 11 & 17 & 11 & 45 & 56 & 72 & 68 & 63 & 14 & 11 & 7 & 15 & 8 & 47 & 55 & 52 & 44 & 64 \\
\hline Cáncer próstata & 1 & 7 & 7 & 6 & 0 & 50 & 69 & 61 & 50 & 53 & 0 & 0 & 0 & 0 & 0 & 0 & 0 & 0 & 0 & 0 \\
\hline Sida & 5 & 11 & 23 & 10 & 29 & 0 & 0 & 0 & 1 & 0 & 3 & 2 & 5 & 10 & 9 & 0 & 1 & 0 & 0 & 0 \\
\hline Diabetes & 2 & 7 & 2 & 6 & 4 & 12 & 35 & 31 & 30 & 28 & 2 & 2 & 1 & 2 & 3 & 32 & 40 & 51 & 44 & 51 \\
\hline Cardiopatía isquémica & 44 & 42 & 37 & 54 & 49 & 155 & 219 & 152 & 150 & 184 & 11 & 3 & 7 & 7 & 6 & 120 & 252 & 151 & 133 & 129 \\
\hline Enf. cerebrovascular & 6 & 10 & 10 & 7 & 10 & 95 & 147 & 121 & 107 & 102 & 4 & 4 & 3 & 6 & 9 & 149 & 206 & 152 & 145 & 165 \\
\hline EPOC & 1 & 2 & 5 & 7 & 5 & 65 & 107 & 120 & 88 & 120 & 0 & 1 & 0 & 2 & 1 & 18 & 42 & 32 & 27 & 34 \\
\hline Cirrosis & 8 & 7 & 16 & 15 & 15 & 8 & 14 & 17 & 17 & 17 & 2 & 2 & 5 & 0 & 6 & 0 & 7 & 12 & 9 & 11 \\
\hline Accidentes tráfico & 23 & 29 & 22 & 23 & 22 & 8 & 8 & 17 & 11 & 8 & 10 & 12 & 6 & 8 & 3 & 5 & 8 & 5 & 8 & 7 \\
\hline Sobredosis & 3 & 3 & 12 & 8 & 24 & 0 & 0 & 0 & 0 & 0 & 0 & 2 & 2 & 0 & 4 & 0 & 0 & 0 & 0 & 1 \\
\hline Caídas accidentales & 1 & 1 & 7 & 6 & 12 & 13 & 7 & 6 & 7 & 12 & 1 & 1 & 0 & 1 & 0 & 14 & 10 & 15 & 8 & 11 \\
\hline Suicidio & 21 & 26 & 26 & 14 & 23 & 6 & 6 & 10 & 8 & 6 & 19 & 3 & 8 & 5 & 12 & 0 & 1 & 1 & 3 & 1 \\
\hline Otras causas & 141 & 131 & 157 & 144 & 187 & 510 & 776 & 656 & 576 & 607 & 90 & 70 & 83 & 75 & 110 & 737 & 1059 & 950 & 755 & 747 \\
\hline
\end{tabular}

\begin{tabular}{|c|c|c|c|c|c|c|c|c|c|c|c|c|c|c|c|c|c|c|c|c|}
\hline \multirow{3}{*}{$\begin{array}{l}\text { Quintiles del índice } \\
\text { socioeconómico }\end{array}$} & \multicolumn{10}{|c|}{ Hombres } & \multicolumn{10}{|c|}{ Mujeres } \\
\hline & \multicolumn{5}{|c|}{$\leq 65$ años } & \multicolumn{5}{|c|}{$>65$ años } & \multicolumn{5}{|c|}{$\leq 65$ años } & \multicolumn{5}{|c|}{$>65$ años } \\
\hline & Q1 & Q2 & Q3 & Q4 & Q5 & Q1 & Q2 & Q3 & Q4 & Q5 & Q1 & Q2 & Q3 & Q4 & Q5 & Q1 & Q2 & Q3 & Q4 & Q5 \\
\hline Total & 236 & 306 & 284 & 300 & 416 & 758 & 1132 & 1140 & 1000 & 1065 & 120 & 114 & 105 & 127 & 156 & 750 & 1212 & 1237 & 897 & 1071 \\
\hline Cáncer pulmón & 23 & 35 & 33 & 43 & 42 & 45 & 68 & 73 & 53 & 89 & 7 & 7 & 1 & 3 & 4 & 8 & 15 & 9 & 9 & 11 \\
\hline Cáncer mama & & & & & & & & & & & 16 & 10 & 15 & 15 & 19 & 18 & 21 & 36 & 21 & 31 \\
\hline Cáncer estómago & 7 & 9 & 8 & 5 & 13 & 11 & 27 & 20 & 31 & 24 & 2 & 0 & 8 & 2 & 2 & 20 & 19 & 9 & 16 & 12 \\
\hline Cáncer colorrectal & 10 & 14 & 15 & 10 & 12 & 37 & 55 & 42 & 43 & 37 & 6 & 4 & 6 & 5 & 9 & 20 & 33 & 51 & 26 & 32 \\
\hline Cáncer próstata & 7 & 1 & 2 & 2 & 3 & 34 & 57 & 55 & 46 & 41 & & & & & & & & & & \\
\hline Sida & 3 & 15 & 15 & 12 & 28 & 0 & 0 & 0 & 2 & 1 & 3 & 1 & 1 & 2 & 7 & 0 & 0 & 0 & 0 & 0 \\
\hline Diabetes & 3 & 3 & 2 & 4 & 3 & 18 & 21 & 19 & 12 & 24 & 0 & 0 & 2 & 1 & 2 & 22 & 36 & 50 & 31 & 33 \\
\hline Cardiopatía isquémica & 22 & 33 & 22 & 25 & 22 & 90 & 96 & 133 & 100 & 98 & 3 & 5 & 3 & 3 & 8 & 55 & 94 & 96 & 62 & 93 \\
\hline Enf. cerebrovascular & 10 & 10 & 9 & 10 & 20 & 67 & 98 & 82 & 80 & 82 & 4 & 7 & 7 & 8 & 9 & 66 & 165 & 161 & 102 & 115 \\
\hline EPOC & 2 & 0 & 3 & 3 & 8 & 37 & 59 & 74 & 49 & 88 & 0 & 1 & 2 & 0 & 1 & 15 & 20 & 17 & 18 & 24 \\
\hline Cirrosis & 6 & 10 & 8 & 6 & 18 & 11 & 10 & 20 & 16 & 17 & 2 & 3 & 2 & 2 & 4 & 1 & 10 & 11 & 6 & 16 \\
\hline Accidentes tráfico & 14 & 30 & 20 & 23 & 43 & 3 & 7 & 2 & 5 & 11 & 10 & 6 & 5 & 10 & 11 & 2 & 8 & 9 & 3 & 5 \\
\hline Sobredosis & 2 & 0 & 4 & 6 & 6 & 0 & 0 & 0 & 0 & 0 & 1 & 0 & 2 & 0 & 0 & 0 & 0 & 0 & 0 & 0 \\
\hline Caídas accidentales & 6 & 3 & 4 & 4 & 5 & 1 & 14 & 9 & 8 & 6 & 0 & 1 & 0 & 1 & 0 & 5 & 18 & 13 & 6 & 6 \\
\hline Suicidio & 15 & 9 & 11 & 17 & 17 & 7 & 8 & 13 & 5 & 14 & 2 & 6 & 3 & 7 & 6 & 1 & 2 & 7 & 1 & 5 \\
\hline Otras causas & 89 & 100 & 108 & 111 & 153 & 329 & 511 & 492 & 460 & 430 & 58 & 60 & 41 & 61 & 71 & 426 & 661 & 664 & 504 & 594 \\
\hline
\end{tabular}


Tabla 4. Asociación entre el índice de privación socioeconómico y la mortalidad. RR comparando la mortalidad en las unidades censales del primer y último quintil. Pamplona y Logroño (1996-2007)

\begin{tabular}{|c|c|c|c|c|c|c|c|c|}
\hline \multicolumn{9}{|c|}{ Pamplona } \\
\hline & \multicolumn{4}{|c|}{ Hombres } & \multicolumn{4}{|c|}{ Mujeres } \\
\hline & \multicolumn{2}{|c|}{ 0-64 años } & \multicolumn{2}{|c|}{$\geq 65$ años } & \multicolumn{2}{|c|}{ 0-64 años } & \multicolumn{2}{|c|}{$\geq 65$ años } \\
\hline & $\mathbf{R R}$ & (IC 95\%) & $\mathbf{R R}$ & (IC 95\%) & $\mathbf{R R}$ & (IC 95\%) & $\mathbf{R R}$ & (IC 95\%) \\
\hline Todas las causas & 1,61 & $(1,32-1,92)$ & 1,23 & $(1,09-1,36)$ & 1,44 & $(1,18-1,73)$ & 1,11 & $(0,97-1,25)$ \\
\hline Cáncer de pulmón & 1,42 & $(0,97-1,99)$ & 1,34 & $(1,02-1,73)$ & 6,09 & $(2,02-14,90)$ & 0,48 & $(0,15-1,05)$ \\
\hline Cáncer de mama & & & & & 0,91 & $(0,48-1,52)$ & 0,87 & $(0,47-1,46)$ \\
\hline Cáncer de estómago & 3,02 & $(1,17-6,54)$ & 1,19 & $(0,70-1,86)$ & 3,24 & $(0,19-15,07)$ & 0,94 & $(0,49-1,59)$ \\
\hline Cáncer colorrectal & 0,72 & $(0,19-1,75)$ & 1,48 & $(0,89-2,31)$ & 0,71 & $(0,16-1,80)$ & 1,21 & $(0,71-1,91)$ \\
\hline Cáncer de próstata & & & 1,20 & $(0,73-1,84)$ & & & & \\
\hline Sida & 8,06 & $(2,55-20,53)$ & & & 6,21 & $(1,04-24,53)$ & & \\
\hline Diabetes & 6,49 & $(0,37-29,90)$ & 2,48 & $(1,12-4,78)$ & 3,77 & $(0,48-15,88)$ & 1,62 & $(0,92-2,62)$ \\
\hline Cardiopatía isquémica & 1,36 & $(0,89-1,98)$ & 1,23 & $(0,93-1,60)$ & 0,69 & $(0,09-2,11)$ & 1,20 & $(0,88-1,59)$ \\
\hline Enf. cerebrovascular & 2,83 & $(0,61-8,86)$ & 1,15 & $(0,87-1,48)$ & 5,48 & $(0,71-21,70)$ & 1,23 & $(0,94-1,57)$ \\
\hline EPOC & & & 1,89 & $(1,34-2,56)$ & & & 2,05 & $(1,09-3,52)$ \\
\hline Cirrosis hepática & 2,69 & $(1,05-5,75)$ & 2,32 & $(0,75-5,54)$ & 19,01 & $(1,74-99,31)$ & & \\
\hline Accidentes de tráfico & 1,11 & $(0,60-1,85)$ & 1,26 & $(0,36-3,15)$ & 0,46 & $(0,056-1,50)$ & 2,61 & $(0,36-9,92)$ \\
\hline Sobredosis & 11,94 & $(4,24-34,25)$ & & & & & & \\
\hline Caídas accidentales & & & 0,94 & $(0,31-2,08)$ & & & 0,89 & $(0,37-1,74)$ \\
\hline Suicidio & 1,28 & $(0,71-2,12)$ & 1,22 & $(0,34-3,05)$ & 0,81 & $(0,19-2,11)$ & & \\
\hline Otras causas & 1,50 & $(1,13-1,95)$ & 1,24 & $(1,06-1,43)$ & 1,62 & $(1,16-2,19)$ & 1,12 & $(0,98-1,26)$ \\
\hline \multicolumn{9}{|c|}{ Logroño } \\
\hline & \multicolumn{4}{|c|}{ Hombres } & \multicolumn{4}{|c|}{ Mujeres } \\
\hline & \multicolumn{2}{|c|}{ 0-64 años } & \multicolumn{2}{|c|}{$\geq 65$ años } & \multicolumn{2}{|c|}{ 0-64 años } & \multicolumn{2}{|c|}{$\geq 65$ años } \\
\hline & $\mathbf{R R}$ & (IC 95\%) & $\mathbf{R R}$ & (IC 95\%) & $\mathbf{R R}$ & (IC 95\%) & $\mathbf{R R}$ & (IC 95\%) \\
\hline Todas las causas & 1,77 & $(1,55-2,01)$ & 1,04 & $(0,97-1,12)$ & 1,48 & $(1,23-1,76)$ & 1,14 & $(1,05-1,23)$ \\
\hline Cáncer de pulmón & 1,84 & $(1,26-2,61)$ & 1,44 & $(1,11-1,85)$ & 0,81 & $(0,29-1,69)$ & 1,08 & $(0,53-1,96)$ \\
\hline Cáncer de mama & & & & & 1,44 & $(0,87-2,25)$ & 1,32 & $(0,86-1,97)$ \\
\hline Cáncer de estómago & 1,90 & $(0,95-3,51)$ & 1,61 & $(0,95-2,62)$ & 1,45 & $(0,21-4,46)$ & 0,47 & $(0,27-0,75)$ \\
\hline Cáncer colorrectal & 1,23 & $(0,65-2,14)$ & 0,74 & $(0,53-1,01)$ & 1,87 & $(0,84-3,68)$ & 1,26 & $(0,83-1,84)$ \\
\hline Cáncer de próstata & 0,45 & $(0,14-0,99)$ & 0,90 & $(0,64-1,23)$ & & & & \\
\hline Sida & 10,75 & $(4,42-24,23)$ & & & 3,28 & $(1,14-7,93)$ & & \\
\hline Diabetes & 1,10 & $(0,28-2,86)$ & 1,01 & $(0,63-1,53)$ & & & 1,22 & $(0,81-1,76)$ \\
\hline Cardiopatía isquémica & 1,04 & $(0,67-1,55)$ & 0,81 & $(0,65-0,99)$ & 3,33 & $(1,19-7,96)$ & 1,35 & $(1,05-1,70)$ \\
\hline Enf. cerebrovascular & 2,02 & $(1,14-3,37)$ & 0,92 & $(0,72-1,15)$ & 2,81 & $(1,14-6,11)$ & 1,40 & $(1,12-1,74)$ \\
\hline EPOC & 4,44 & $(2,19-12,23)$ & 1,78 & $(1,34-2,34)$ & & & 1,32 & $(0,81-2,04)$ \\
\hline Cirrosis hepática & 3,26 & $(1,63-6,09)$ & 1,16 & $(0,66-1,94)$ & 2,78 & $(0,98-8,06)$ & 15,32 & $(0,77-55,31)$ \\
\hline Accidentes de tráfico & 3,08 & $(1,98-4,67)$ & 2,90 & $(1,09-6,79)$ & 1,26 & $(0,65-2,20)$ & 2,20 & $(0,00-6,27)$ \\
\hline Sobredosis & 3,48 & $(2,10-9,77)$ & & & & & & \\
\hline Caídas accidentales & 0,89 & $(0,34-1,87)$ & & & & & 1,05 & $(0,41-2,23)$ \\
\hline Suicidio & 1,20 & $(0,71-1,91)$ & 1,55 & $(0,78-2,84)$ & 3,96 & $(2,30-11,12)$ & & \\
\hline Otras causas & 1,77 & $(1,44-2,16)$ & 0,98 & $(0,88-1,09)$ & 1,39 & $(1,07-1,77)$ & 1,12 & $(1,02-1,24)$ \\
\hline
\end{tabular}


Los menores de 65 años presentaron una mayor mortalidad por sida en las zonas más desfavorecidas socio-económicamente, oscilando los RR de mortalidad de los quintiles con mayor privación entre 3,28 y 10,75 , siendo en todos los casos estadísticamente significativos. También la mortalidad por cirrosis y por sobredosis de drogas mostró un gradiente positivo. Para el cáncer de pulmón se encontró una asociación positiva entre los hombres de ambas ciudades así como en las mujeres menores de 65 años de Pamplona. El cáncer de estómago presentó RR de mortalidad más elevados en las zonas más desfavorecidas aunque sólo eran estadísticamente significativos entre los hombres de Pamplona. La cardiopatía isquémica en mujeres y la enfermedad cerebrovascular en ambos sexos mostraron una asociación positiva en Logroño. Finalmente señalar que entre los varones de 0-64 años de Logroño se observó un RR de mortalidad por accidente de tráfico más elevado en las zonas pertenecientes al quintil más desfavorecido.

En los hombres mayores de 65 años, se encontró asociación positiva entre mortalidad y privación para la EPOC y el cáncer de pulmón entre los hombres de ambas ciudades. También se encontró una asociación positiva para la mortalidad por diabetes en Pamplona y los accidentes de tráfico en Logroño. Entre las mujeres mayores de 65 años, sólo la mortalidad por EPOC en Pamplona y la mortalidad por enfermedad isquémica del corazón y enfermedad cerebrovascular en Logroño mostraron una asociación positiva estadísticamente significativa con la privación socioeconómica.

\section{DISCUSIÓN}

Se evidenció una asociación entre la mortalidad total y el índice de privación tanto en hombres como en mujeres. Se observó un riesgo de mortalidad total mayor en aquellas unidades censales con peores indicadores socioeconómicos. El análisis desagregado por edad muestra una asociación mayor entre los hombres menores de 65 años. También se observó relación entre la mortalidad por algunas causas especí- ficas e índice de privación. En mujeres, la asociación entre el índice socioeconómico y la mortalidad ha sido menor.

Otros estudios desarrollados en ciudades españolas ${ }^{13}$ y en la Comunidad Autónoma del País Vasco (CAPV) ${ }^{9}$ muestran algunos resultados coincidentes con nuestro estudio. En la CAPV, en el análisis desagregado por edad se encontró que, como en Pamplona y Logroño, la asociación entre privación socioeconómica y mortalidad es mayor entre los jóvenes y entre los hombres. Estas diferencias podrían explicarse por la distinta historia laboral de hombres y mujeres, así como por los distintos estilos de vida según el sexo 9 .

En relación a las causas de mortalidad relacionadas con el índice de privación y en la línea de otros estudios, las mayores diferencias se observaron para causas como sobredosis por drogas, sida, EPOC, cirrosis hepática y cáncer de pulmón. En España, el estudio MEDEA desarrollado en el periodo 1996-2003 encontró que entre los hombres, cuatro causas específicas de muerte (cáncer de pulmón, cardiopatía isquémica, enfermedades respiratorias y cirrosis) estaban asociadas positivamente con la privación en la mayoría de las ciudades (a mayor privación, mayor mortalidad). Entre las mujeres tres causas presentaban asociación, dos positiva (diabetes y cirrosis) y una negativa (cáncer de pulmón). Los resultados de Pamplona y Logroño coinciden en buena medida aunque se observan algunas diferencias como la asociación positiva del cáncer de pulmón entre las mujeres jóvenes de Pamplona, es decir mayor riesgo de muerte por esta causa en las zonas con mayor privación socioeconómica. Dado que los datos de nuestro estudio se refieren a un periodo más reciente podrían estar reflejando una prevalencia de tabaquismo mayor en las mujeres residentes en las zonas más desfavorecidas.

Coincide con lo encontrado en otros estudios que la magnitud de las desigualdades ha sido mayor para las causas de muerte que, como la EPOC, sobredosis por drogas, cirrosis o el cáncer de pulmón, están directamente relacionadas con el consumo de tabaco o alcohol. Destacan tam- 
bién por su magnitud las desigualdades en la mortalidad por sida y sobredosis. Ambas causas de muerte están muy ligadas al consumo de drogas por vía parenteral y a la marginalidad, en las que además de la privación material desempeñan un papel relevante los factores psicosociales ligados a la desestructuración social ${ }^{13}$.

En Navarra otro estudio que empleaba el barrio de residencia como unidad de análisis exploró las diferencias en la mortalidad relacionada con el nivel socioeconómico en población menor de 75 años del periodo 1985-1989. A cada barrio se le asignó un nivel socioeconómico en función de los siguientes indicadores del censo: situación laboral, nivel de estudios, profesión, y nivel de equipamientos del hogar e ingresos familiares. Se llevó a cabo una regresión lineal entre las tasas ajustadas de mortalidad y el estatus socioeconómico y se concluyó que, para los hombres, se observaba una clara tendencia creciente de la mortalidad en relación al empeoramiento del estatus socioeconómico del barrio ${ }^{17}$.

Destacar igualmente, el estudio realizado sobre mortalidad y ocupación en los varones de Navarra ${ }^{18}$ que evidenció diferencias en la mortalidad en ciertas actividades profesionales, tanto de la mortalidad total como en la mortalidad específica por algunas causas.

En el año 2009, entre más de 200 regiones de Europa, la esperanza de vida al nacer de las mujeres de Navarra y La Rioja se situaba en el primer y cuarto lugar, con 86,6 y 86,1 años respectivamente. La esperanza de vida de los hombres que fue de 80 años en ambas regiones, se encontraba entre las 15 más altas en un ranking liderado por la región de Ticino (Italia) con 80,7 años ${ }^{19}$. Los datos presentados en este trabajo son un complemento y no empañan el buen dato general pero este tipo de estudios deben ser considerados para orientar las políticas de salud y también las políticas sociales con el objetivo último de disminuir las desigualdades.

El estudio presenta algunas limitaciones. Así como señalan los autores del estudio MEDEA llevado a cabo por Borrell y col en 2010, en el que se comparaban los datos de 11 ciudades españolas, las asociaciones más importantes se encuentran en las ciudades más grandes, algo que puede estar relacionado con dos aspectos. En primer lugar, la existencia de grandes desigualdades socioeconómicas en estas ciudades, áreas de concentración con alta marginación y peor salud. En segundo lugar, por el mayor número de secciones censales, hay una mayor potencia estadística. En este sentido, la falta de resultados significativos en las ciudades más pequeñas no necesariamente significa que en esas ciudades el efecto de la privación de mortalidad sea insignificante, ya que podría deberse simplemente a una falta de potencia estadística ${ }^{13}$. En nuestro estudio la falta de potencia estadística nos ha llevado a analizar los datos agregados de 12 años. La utilización de los datos socioeconómicos de un año para estimar los riesgos de un periodo de 12 años puede ser discutible pero hay que tener en cuenta que los periodos de latencia para la mayoría de las causas de muerte estudiadas son largos (cáncer, cirrosis, enfermedades cardiovasculares o diabetes).

Por otro lado podemos señalar en primer lugar el éxito de la geocodificación de los datos en ambas ciudades, superando al 98\% de las defunciones objeto de estudio. Además del gradiente existente en la mortalidad y el índice de privación, se ha podido constatar la gran similitud existente en el riesgo de muerte entre ambas poblaciones dependiendo de la pertenencia a una zona más o menos privilegiada. Es decir, el exceso de mortalidad encontrado en ambas ciudades es muy similar lo que apunta a que probablemente las causas que subyacen sean parecidas

Los resultados obtenidos en este estudio pueden ayudar en un futuro en la planificación de recursos sanitarios y la puesta en marcha de programas de prevención que ayuden a la disminución de las desigualdades en salud.

\section{BIBLIOGRAFÍA}

1. Stringhini S, Sabia $S$, Shipley M, Brunner E, Nabi $\mathrm{H}$, KIVIMAKI $\mathrm{M}$ et al. Association of socioeconomic position with health behaviors and mortality. JAMA 2010; 303: 1159-1166. 
2. Hummer RA, Rogers RG, Eberstein IW. Sociodemographic differentials in adult mortality: a review of analytic approaches. Popul Dev Rev 1998; 24: 553-578.

3. Ruiz-Ramos M, Sánchez J, Garrucho G, Viciana F. Desigualdades en mortalidad en la ciudad de Sevilla. Gac Sanit 2004; 18: 16-23.

4. Mackenbach JP, Stirbu I, Roskam AJ, SchaAp MM, Menvielle G, Leinsalu M et al. Socioeconomic inequalities in health in 22 European countries. N Engl J Med 2008; 358: 2468-881.

5. Gallo V, Mackenbach JP, Ezzati M, Menvielle G, Kunst AE, Rohrmann $S$ et al. Social Inequalities and Mortality in Europe. Results from a Large Multi-National Cohort, 2012 PLoS ONE 7(7): e39013. doi:10.1371/journal.pone.0039013.

6. CARStairs V. Small area analysis and health service research. Community Med 1981; 3: 131-139.

7. CARstairs V, Morris R. Deprivation and mortality: an alternative to social class? Community Med 1989; 11: 210-219.

8. CARstairs V, Morris R. Deprivation: explaining differences in mortality between Scotland and England and Wales. BMJ 1989; 299: 886889.

9. Esnaola S, Aldasoro E, Ruiz R, Audicana C, Pérez Y, Calvo M. Desigualdades socioeconómicas en la mortalidad en la Comunidad Autónoma del País Vasco. Gac Sanit 2006; 20: 16-24.

10. Macintyre S, Ellaway A. Neighborhoods and Health: An Overview. En: Kawachi BL, Oxford I. (Eds), University Press, Oxford, 2003: 20-42.

11. Macintyre S, Ellaway A, Cummins S. Place effects on health: how can we conceptualise, operationalise and measure them? Soc Sci Med 2002; 55: 125-139.
12. KJellstrom, T. Our cities, our health, our future. WHO Commission on socialdeterminants of health from the knowledge network on urban settings acting on social determinants for health equity in urban settings, Kobe (Japan), 2008.

13. Borrell C, Marí-Dell'olmo M, Serral G, Martínez-Beneito M, Gotsens M, MEDEA Members: Inequalities in mortality in small areas of eleven Spanish cities (the multicenter MEDEA project). Health Place 2010; 16:703-711.

14. Domínguez-Berjón MF, Borrell C, Cano-Serral G, Esnaola S, Nolasco A, Pasarín MA et al. Construcción de un índice de privación a partir de datos censales en grandes ciudades españolas (Proyecto MEDEA). Gac Sanit 2008; 22: 179-187.

15. ChIANG CL. The life table and its applications. Malabar: Robert E. Krieger; 1984.

16. Besag J, York J, Mollie A: Bayesian image restoration, with two applications in spatial statistics. Ann Inst Statist Math 1991; 43: 1-59.

17. Casi Casanellas A, Moreno Iribas C. Desigualdad ante la muerte: Estudio comparativo entre comunidades de Navarra en el segmento de población de 25 a 74 años. Aten Primaria 1992; 10: 543-548.

18. Ugarte MD, Artieda L, Ibañez B, Militino AF, Lezaun M, Lopez-Sagaseta M, Moreno-Iribas C. A cohort study to estimate occupational mortality risks in Navarra. Eur J Public Health 2005; 5: 305-312.

19. Population statistics at regional level - Statistics Explained (2013/1/4) Disponible en: http://epp.eurostat.ec.europa.eu/statistics_ explained/index.php/Population_statistics_ at_regional_level. 